\title{
A NEW MAGNETO-OPTICAL EFFECT IN RAMAN SPECTROSCOPY
}

\author{
David L. ANDREWS \\ School of Chemical Sciences, University of East Anglia, Norwich, NR4 7TJ, UK
}

Received 2 September 1986

\begin{abstract}
It is well known that the application of a static magnetic field can result in a change in both Raman spectral intensities and line positions. This effect is generally associated with a removal of spin degeneracy, and leads to a manifestation of chiral discrimination in both optically active and inactive samples. However, other magnetic field effects can be conferred upon the Raman process by virtue of direct magneto-optical interactions. It is here shown that for molecules belonging to one of the cubic or icosahedral point groups, any polarised Raman transition associated with a totally symmetric vibration becomes depolarised on application of a magnetic field directed along the usual right-angled direction of observation. Hence with a suitable polarisation filter, the corresponding Raman line can be "switched" into the spectrum by application of the field.
\end{abstract}

\section{Introduction}

The effect of a static magnetic field on optical processes has a long and honourable history, dating back to Michael Faraday's discovery of field-induced optical rotation in 1845 [1]. With magnetic flux densities of $10 \mathrm{~T}$ and more now available using superconducting magnets, some of the weaker magneto-optical effects are increasingly coming under scrutiny. In the case of Raman scattering, most attention has been focused on the field-induced vibrational optical activity which can be measured by circular differential resonance Raman spectroscopy [2]. In particular, polarisation anomalies associated with antisymmetric scattering have been demonstrated to arise from the removal of electronic spin degeneracy, and can thus be used to probe Zeeman splitting in the electronic ground state [3].

In this paper, a novel magneto-optical effect is described in which the magnetic field plays a direct role in inducing a vibrational Raman transition; an analogous effect takes place in two-photon absorption spectroscopy [4]. The magneto-optical Raman effect described here is one which should be manifest for any totally symmetric vibration in a molecule belonging to one of the cubic or icosahedral point groups. Raman lines associated with such vibrations are normally polarised, and hence disappear from the spectrum if a depolarising filter is inserted between the sample and the detector. However, application of a static field is shown to result in a depolarisation of the Raman signal, so that such vibrations become active in the depolarised spectrum. One particularly notable feature of this effect is that it does not involve the splitting of states with spin degeneracy.

\section{Calculation of the Raman scattering intensity}

We begin by defining terms in which to describe a general Raman transition involving annihilation of a laser photon of wave vector $\boldsymbol{k}$, circular frequency $\omega=c|\boldsymbol{k}|$ and polarisation vector $\boldsymbol{e}$, together with creation of a photon of wave vector $\boldsymbol{k}^{\prime}$, circular frequency $\omega^{\prime}=c\left|\boldsymbol{k}^{\prime}\right|$ and polarisation vector $\boldsymbol{e}^{\prime}$. The accompanying molecular transition is represented by $|n\rangle \leftarrow|m\rangle$. The Raman scattering intensity in the 
presence of a static magnetic field $\boldsymbol{B}$ can be calculated using second-order time-dependent perturbation theory, using dressed basis states given by

$$
\left|r^{\prime}\right\rangle=|r\rangle-\sum_{r \neq s}\left(\boldsymbol{m}^{s r} \cdot \boldsymbol{B}\right) E_{r s}^{-1}|s\rangle
$$

where $E_{r s}$ represents the difference in the zeroth-order energies $E_{r}-E_{s}$, and $\boldsymbol{m}^{s r}$ is the magnetic dipole transition moment for the transition $|s\rangle \leftarrow|r\rangle$. The states $|m\rangle,|r\rangle,|s\rangle$ and $|n\rangle$ are to be regarded as vibronic states; in the case where the Raman transition is purely vibrational, then $|m\rangle$ and $|n\rangle$ are vibrational levels belonging to the electronic ground state. The Raman intensity $I^{\prime}\left(k^{\prime}\right)$, defined as the power emitted per unit solid angle about the direction $\boldsymbol{k}^{\prime}$, follows directly from the Fermi rule expression for the scattering rate, and may be written as;

$$
I^{\prime}=\left(k^{\prime 4} I_{0} / 16 \pi^{2} \epsilon_{0}^{2}\right)\left\langle\left|M_{n m}^{\prime}\right|^{2}\right\rangle
$$

where $I_{0}$ is the laser irradiance, and $M_{n m}^{\prime}$ is the reduced matrix element; the angular brackets in eq. (2) denote the rotational average appropriate for a fluid sample. Full details of the quantum electrodynamical methods used for the calculation of the matrix element are discussed in detail elsewhere [5]. Using the implied summation convention for repeated indices, the result is expressible as follows;

$$
M_{n m}^{\prime}=M^{\prime}(\alpha)+M^{\prime}(\chi)+\cdots=\left\{\alpha_{i j}^{n m}(\omega) e_{i}^{\prime} e_{j}+\chi_{i j k}^{n m}(\omega) e_{i}^{\prime} e_{j} B_{k}+\cdots\right\}
$$

where $\alpha_{i j}^{n m}(\omega)$ is the usual Raman scattering tensor, and $\chi_{i j k}^{n m}(\omega)$ is a third-rank molecular tensor defined by

$$
\begin{aligned}
\chi_{i j k}^{n m}= & \sum_{r, s}\left[\frac{\mu_{i}^{n s} \mu_{j}^{s r} m_{k}^{r m}}{\left(E_{s n}+\hbar \omega\right) E_{r m}}+\frac{\mu_{i}^{n s} m_{k}^{s r} \mu_{j}^{r m}}{\left(E_{s n}+\hbar \omega\right)\left(E_{r n}+\hbar \omega\right)}+\frac{m_{k}^{n s} \mu_{i}^{s r} \mu_{j}^{r m}}{E_{s n}\left(E_{r n}+\hbar \omega\right)}+\frac{\mu_{j}^{n s} \mu_{i}^{s r} m_{k}^{r m}}{\left(E_{s m}-\hbar \omega\right) E_{r m}}\right. \\
& \left.+\frac{\mu_{j}^{n s} m_{k}^{s r} \mu_{i}^{r m}}{\left(E_{s m}-\hbar \omega\right)\left(E_{r m}-\hbar \omega\right)}+\frac{m_{k}^{n s} \mu_{j}^{s r} \mu_{i}^{r m}}{E_{s n}\left(E_{r m}-\hbar \omega\right)}\right],
\end{aligned}
$$

where $\mu^{s r}$ denotes an electric dipole transition moment.

The leading contribution to the Raman scattering intensity given by eq. (2) comes from the field-independent term in eq. (3). To obtain a result applicable to a fluid medium, it is necessary to effect a rotational average to account for the random molecular orientations [6]. The following result is then obtained for the intensity of depolarised scattering ( $e^{\prime}$ perpendicular to $e$ ):

$$
I^{\prime}=\left(k^{\prime 4} I_{0} / 480 \pi^{2} \epsilon_{0}^{2}\right)\left(4 \alpha_{\lambda \mu}^{n m} \bar{\alpha}_{\lambda \mu}^{n m}-\alpha_{\lambda \lambda}^{n m} \bar{\alpha}_{\mu \mu}^{n m}-\alpha_{\lambda \mu}^{n m} \bar{\alpha}_{\mu \lambda}^{n m}\right)
$$

In order to clarify the selection rules, it is useful to recast this equation in terms of irreducible cartesian tensors. The irreducible parts of the Raman tensor are as follows:

$$
\begin{aligned}
& \alpha_{\lambda \mu}^{n m}=\alpha_{\lambda \mu}^{(0+)}+\alpha_{\lambda \mu}^{(1+)}+\alpha_{\lambda \mu}^{(2+)}, \\
& \alpha_{\lambda \mu}^{(0+)}=\frac{1}{3} \delta_{\lambda \mu} \alpha_{\nu \nu}^{n m}, \\
& \alpha_{\lambda \mu}^{(1+)}=\frac{1}{2} \epsilon_{\lambda \mu \nu}\left(\epsilon_{o \pi \nu} \alpha_{o \pi}^{n m}\right), \\
& \alpha_{\lambda \mu}^{(2+)}=\frac{1}{2}\left(\alpha_{\lambda \mu}^{n m}+\alpha_{\mu \lambda}^{n m}\right)-\frac{1}{3} \delta_{\lambda \mu} \alpha_{\nu \nu}^{n m},
\end{aligned}
$$

where the $\epsilon$ denotes the Levi-Civita antisymmetric tensor. The three irreducible terms given by eqs. (7)-(9) are simply the trace, the antisymmetric part and the symmetric part of the Raman tensor, respectively, embedded in second-rank tensor space. Contracted with themselves, these are related to the more familiar 
Raman parameters $\alpha^{2}, \delta^{2}$ and $\gamma^{2}[7]$ by the relations $\alpha_{\lambda \mu}^{(0+)} \bar{\alpha}_{\lambda \mu}^{(0+)}=3 \alpha^{2} ; \alpha_{\lambda \mu}^{(1+)} \bar{\alpha}_{\lambda \mu}^{(1+)}=\frac{2}{3} \delta^{2} ; \alpha_{\lambda \mu}^{(2+)} \bar{\alpha}_{\lambda \mu}^{(2+)}=\frac{2}{3} \gamma^{2}$. It is well known that the antisymmetric contribution (weight $1+$ ) is vanishingly small for vibrational Raman transitions if the excitation frequency is well away from an optical absorption band; however, the term is retained in the development of the theory below so as to additionally include the possibility of application to either near-resonance or, equally, an electronic Raman transition.

In terms of the irreducible parts of the scattering tensor, the result of eq. (5) is now expressed as;

$$
I^{\prime}=\left(k^{\prime 4} I_{0} / 480 \pi^{2} \epsilon_{0}^{2}\right)\left(5 \alpha_{\lambda \mu}^{(1+)} \bar{\alpha}_{\lambda \mu}^{(1+)}-3 \alpha_{\lambda \mu}^{(2+)} \bar{\alpha}_{\lambda \mu}^{(2+)}\right) .
$$

The important feature of this result is that although in general it contains tensor components of both weight $1+$ and weight $2+$ character, there is no weight $0+$ term.

In the presence of the magnetic field, the leading correction to eq. (10) comes from the cross-terms involving $M^{\prime}(\alpha) \bar{M}^{\prime}(\chi)$ and its complex conjugate. If the field is oriented in the direction defined by $\boldsymbol{e} \times \boldsymbol{e}^{\prime}$, then the rotational average of these two cross-terms gives a vanishing result. However the correction tcrm next largest in magnitude, which results from $\left|M^{\prime}(\chi)\right|^{2}$, persists after rotational averaging and gives

$$
\begin{aligned}
I^{\prime}= & \left(k^{\prime 4} B^{2} I_{0} / 3360 \pi^{2} \epsilon_{0}^{2}\right)\left[16 \chi_{\lambda \mu \nu} \bar{\chi}_{\lambda \mu \nu}-5\left(\chi_{\lambda \lambda \mu} \bar{\chi}_{\nu \nu \mu}+\chi_{\lambda \mu \lambda} \bar{\chi}_{\nu \mu \nu}+\chi_{\lambda \mu \mu} \bar{\chi}_{\lambda \nu \nu}+\chi_{\lambda \mu \nu} \bar{\chi}_{\lambda \nu \mu}\right.\right. \\
& \left.+\chi_{\lambda \mu \nu} \bar{\chi}_{\mu \lambda \nu}+\chi_{\lambda \mu \nu} \bar{\chi}_{\nu \mu \lambda}\right)+2\left(\chi_{\lambda \lambda \mu} \bar{\chi}_{\mu \nu \nu}+\chi_{\lambda \lambda \mu} \bar{\chi}_{\nu \mu \nu}+\chi_{\lambda \mu \lambda} \bar{\chi}_{\mu \nu \nu}+\chi_{\lambda \mu \lambda} \bar{\chi}_{\nu \nu \mu}+\chi_{\lambda \mu \mu} \bar{\chi}_{\nu \lambda \nu}\right. \\
& \left.\left.+\chi_{\lambda \mu \nu} \bar{\chi}_{\nu \lambda \mu}+\chi_{\lambda \mu \mu} \bar{\chi}_{\nu \nu \lambda}+\chi_{\lambda \mu \nu} \bar{\chi}_{\mu \nu \lambda}\right)\right] .
\end{aligned}
$$

Once again this equation is most useful when recast in terms of irreducible tensors. The programme for reduction of a third-rank cartesian tensor into irreducible parts is less well known [8-10], and gives the following results:

$$
\begin{aligned}
\chi_{\lambda \mu \nu}^{n m}= & \chi_{\lambda \mu \nu}^{(0+)}+\chi_{\lambda \mu \nu}^{(1+)}+\chi_{\lambda \mu \nu}^{(2+)}+\chi_{\lambda \mu \nu}^{(3+)}, \\
\chi_{\lambda \mu \nu}^{(0+)}= & \frac{1}{6} \epsilon_{\lambda \mu \nu}\left(\epsilon_{\rho \sigma \tau} \chi_{\rho \sigma \tau}^{n m}\right) \\
\chi_{\lambda \mu \nu}^{(1+)}= & \frac{1}{10}\left[4\left(\delta_{\lambda \mu} \chi_{\rho \rho \nu}^{n m}+\delta_{\lambda \nu} \chi_{\rho \mu \rho}^{n m}+\delta_{\mu \nu} \chi_{\lambda \rho \rho}^{n m}\right)\right. \\
& \left.-\left(\delta_{\lambda \nu} \chi_{\rho \rho \mu}^{n m}+\delta_{\mu \nu} \chi_{\rho \rho \lambda}^{n m}+\delta_{\lambda \mu} \chi_{\rho \nu \rho}^{n m}+\delta_{\mu \nu} \chi_{\rho \lambda \rho}^{n m}+\delta_{\lambda \mu} \chi_{\nu \rho \rho}^{n m}+\delta_{\lambda \nu} \chi_{\mu \rho \rho}^{n m}\right)\right] \\
\chi_{\lambda \mu \nu}^{(2+)}= & \frac{1}{6} \epsilon_{\lambda \mu \tau}\left(2 \epsilon_{\rho \sigma \tau} \chi_{\rho \sigma \nu}^{n m}+2 \epsilon_{\rho \sigma \nu} \chi_{\rho \sigma \tau}^{n m}+\epsilon_{\rho \sigma \tau} \chi_{\nu \rho \sigma}^{n m}+\epsilon_{\rho \sigma \nu} \chi_{\tau \rho \sigma}^{n m}\right) \\
& +\frac{1}{6} \epsilon_{\mu \nu \tau}\left(2 \epsilon_{\rho \sigma \tau} \chi_{\lambda \rho \sigma \sigma}^{n m}+2 \epsilon_{\rho \sigma \lambda} \chi_{\tau \rho \sigma}^{n m}+\epsilon_{\rho \sigma \tau} \chi_{\rho \sigma \lambda}^{n m}+\epsilon_{\rho \sigma \lambda} \chi_{\rho \sigma \tau}^{n m}\right)-\frac{2}{3} \epsilon_{\lambda \mu \nu}\left(\epsilon_{\rho \sigma \tau} \chi_{\rho \sigma \tau}^{n m}\right) \\
\chi_{\lambda \mu \nu}^{(3+)}= & \frac{1}{6}\left(\chi_{\lambda \mu \nu}^{n m}+\chi_{\lambda \nu \mu}^{n m}+\chi_{\mu \lambda \nu}^{n m}+\chi_{\mu \nu \lambda}^{n m}+\chi_{\nu \lambda \mu}^{n m}+\chi_{\nu \mu \lambda}^{n m}\right) \\
& -\frac{1}{15}\left[\delta_{\lambda \mu}\left(\chi_{\rho \rho \nu}^{n m}+\chi_{\rho \nu \rho}^{n m}+\chi_{\nu \rho \rho}^{n m}\right)+\delta_{\lambda \nu}\left(\chi_{\rho \rho \mu}^{n m}+\chi_{\rho \mu \rho}^{n m}+\chi_{\mu \rho \rho}^{n m}\right)+\delta_{\mu \nu}\left(\chi_{\rho \rho \lambda}^{n m}+\chi_{\rho \lambda \rho}^{n m}+\chi_{\lambda \rho \rho}^{n m}\right)\right] .
\end{aligned}
$$

For later reference, it is worth noting that although the weight $0+$ result represents the fully index-antisymmetric part of the tensor $\chi^{n m}$, this does not disappear even for off-resonance vibrational Raman transitions. Using the transformation matrices given in ref. (11), eq. (11) is now reexpressed as follows;

$$
I^{\prime}=\left(k^{\prime 4} B^{2} I_{0} / 3360 \pi^{2} \epsilon_{0}^{2}\right)\left(5 \chi_{\lambda \mu \nu}^{(0+)} \bar{\chi}_{\lambda \mu \nu}^{(0+)}+14 \chi_{\lambda \mu \nu}^{(2+)} \bar{\chi}_{\lambda \mu \nu}^{(2+)}+5 \chi_{\lambda \mu \nu}^{(3+)} \bar{\chi}_{\lambda \mu \nu}^{(3+)}\right)
$$

Comparing the result with the field-independent result, eq. (10), reveals that transitions associated with both weights $0+$ and $3+$ hecome allowed only when the field is present. The significance of this feature can now be explored further by reference to the Raman selection rules. 


\section{Irreducible tensors and selection rules}

The transformation properties of each of the Raman scattering tensors under the operations of the full rotation group are obtained by coupling the representations of the appropriate electric dipole and magnetic dipole moments, and are thus given by:

$$
\begin{aligned}
& D^{(1-)} \otimes D^{(1-)}=D^{(0+)} \oplus D^{(1+)} \oplus D^{(2+)}, \\
& D^{(1-)} \otimes D^{(1-)} \otimes D^{(1+)}=D^{(0+)} \oplus 3 D^{(1+)} \oplus 2 D^{(2+)} \oplus D^{(3+)}
\end{aligned}
$$

for $\alpha_{i j}^{n m}(\omega)$ and $\chi_{i j k}^{n m}(\omega)$ respectively. Tensor components which transform under the totally symmetric representation $D^{(0+)}$ behave as scalars; $D^{(1+)}$ components have the transformation properties of rotations; the $D^{(2+)}$ components transform together as a traceless, index-symmetric axial second-rank tensor, and the $D^{(3+)}$ components as a traceless, fully index-symmetric axial third-rank tensor.

The essential selection rule for Raman transitions is that the product of the representations of the initial and final states must contain an irreducible representation spanned by components of the appropriate transition tensor [12]. In the case of a fundamental vibrational transition, this reduces to a requirement for the irreducible representation of the vibration itself to be spanned by the tensor components. In the particular case of a totally symmetric vibration in a molecule belonging to one of the cubic or icosahedral point groups $\mathrm{T}, \mathrm{T}_{\mathrm{h}}, \mathrm{T}_{\mathrm{d}}, \mathrm{O}, \mathrm{O}_{\mathrm{h}}$, I or $\mathrm{I}_{\mathrm{h}}$, the only tensor components which are not identically zero are those which transform as a scalar, in other words the weight $0+$ components. The absence of any such components from the result given by eq. (6) thus has the well-known implication that any such vibration is inactive in the depolarised Raman spectrum, and the Raman signal is thus regarded as fully polarised [13].

However, the appearance of weight $0+$ components in the field-induced term given by equation (17) means that the magnetic field allows depolarised Raman scattering to occur even for these vibrations. Thus when the Raman signal of a molecule with suitably high symmetry is collected through a polarisation analyser crossed with respect to the pump laser polarisation, a magnetic field can be used to "switch" the totally symmetric vibrations into the spectrum. Examples are afforded by the breathing modes of methane and sulphur hexafluoride.

\section{Discussion}

An estimate can readily be made of the likely magnitude of the effect discussed above. Inspection of the detailed structure of the $\alpha^{n m}$ and $\chi^{n m}$ tensors shows that the field-induced rate given by eq. (17) is smaller than the normal Raman scattering rate by a factor of the order of $\beta^{2}=\left(\boldsymbol{m}^{p q} \cdot \boldsymbol{B} / \Delta E\right)^{2}$, where $\boldsymbol{m}^{p q}$ is a typical magnetic dipole transition moment and $\Delta E$ a typical detuning from resonance. Thus if $\left|m^{p q}\right| \approx 1$ Bohr magneton, $|\boldsymbol{B}| \approx 1 \mathrm{~T}$ and $\Delta E \approx 500 \mathrm{~cm}^{-1}$, the value of $\beta$ is approximately $10^{-3}$, so that the scattering is less intense than a typical Raman signal by a factor of about $10^{-6}$. This is still appreciably more intense than, for example, the typical intensity of a hyper-Raman spectrum, although the necessity for collection of the scattered light through a magnet aperture would impose a further limitation. However, with a magnetic field of $10 \mathrm{~T}$ such as is now commonly available from superconducting magnets, $\beta$ has a value of about $10^{-2}$, making the field-induced intensity only about $10^{-4}$ times smaller than that of a normal Raman signal. This kind of signal level is similar to that associated with the circular differential Raman scattering displayed by optically active molecules, which is well established as an experimental technique [14]. Measurement of the effect would therefore call for the adoption of similar instrumental methods based on electro-optic modulation of the laser beam coupled with phase-sensitive detection. Hence the effect described here should certainly be amenable to experimental verification. 


\section{References}

[1] M. Faraday, Phil. Trans. Roy. Soc. 136 (1846) 1.

[2] L.D. Barron and J. Vrbancich, in: Advances in infrared and Raman spectroscopy, Vol. 12, eds. R.J.H. Clark and R.E. Hester (Wiley, New York, 1985) p. 215.

[3] L.D. Barron, Molecular light scattering and optical activity (Cambridge University Press, Cambridge, 1982).

[4] D.L. Andrews, J. Phys. B19 (1986) L613.

[5] D.P. Craig and T. Thirunamachandran, Molecular quantum electrodynamics (Academic Press, New York, 1984).

[6] D.L. Andrews and T. Thirunamachandran, J. Chem. Phys. 67 (1977) 5026.

[7] D.A. Long, Raman spectroscopy (McGraw-Hill, New York, 1977).

[8] J.A.R. Coope, R.F. Snider and F.R. McCourt, J. Chem. Phys. 43 (1965) 2269.

[9] J. Jerphagnon, Phys. Rev. B2 (1970) 1091.

[10] D.L. Andrews and T. Thirunamachandran, J. Chem. Phys. 68 (1978) 2941.

[11] D.L. Andrews and P.J. Wilkes, J. Chem. Phys. 83 (1985) 2009.

[12] S.F.A. Kettle, Symmetry and structure (Wiley, New York, 1985).

[13] J.A. Koningstein, Introduction to the theory of the Raman effect (Reidel, Dordrecht, 1972).

[14] L.A. Nafie, in: Advances in infrared and Raman spectroscopy, Vol. 11, eds. R.J.H. Clark and R.E. Hester (Wiley, New York, 1985) p. 49. 